\title{
Quantum ground states as equilibrium particle-vacuum interaction states
}

\author{
Harold E. Puthoff [
}

Received: 10 April 2015 / Accepted: 31 July 2015 / Published online: 14 August 2015

(C) The Author(s) 2015. This article is published with open access at Springerlink.com

\begin{abstract}
A remarkable feature of atomic ground states is that they are observed to be radiationless in nature, despite (from a classical viewpoint) typically involving charged particles in accelerated motions. The simple hydrogen atom is a case in point. This universal ground-state characteristic is shown to derive from particle-vacuum interactions in which a dynamic equilibrium is established between radiation emission due to particle acceleration, and compensatory absorption from the zero-point fluctuations of the vacuum electromagnetic field. The result is a net radiationless ground state. This principle constitutes an overarching constraint that delineates an important feature of quantum ground states.
\end{abstract}

Keywords Quantum ground states - Vacuum fluctuations - Particle-vacuum interaction states - Zero-point fluctuations $\cdot$ Harmonic oscillator quantum ground state

\section{Introduction}

One of the apparent paradoxes of quantum theory that students often query is the radiationless nature of atomic ground states. The paradox lies in the fact that, classically, radiation that might be anticipated to occur from accelerated charged-particle motions in atomic ground states is not observed. In the hydrogen atom, for example, the orbiting electron does not radiate its energy away and spiral into the nucleus. The fact that during decades of successful application of quantum theory we have come to take for granted the radiationless feature of these special "bottom-rung" stationary states does not in any way detract from this remarkable property. Fortunately, a rapprochement between classical and quantum viewpoints with regard to ground states is possible.

When addressed in analytical detail it becomes clear wherein the resolution to this apparent paradox lies. It is that one must properly take into account how charged-particle ground-state motions interact with the vacuum, specifically the zero-point fluctuations (ZPFs) of the vacuum electromagnetic field. Although such considerations are not usually invoked in the day-to-day application of quantum theory to ground-state specification, the argument that follows clarifies that the standard quantum formalism with its associated radiationless ground states has its genesis in the dynamics of underlying particle-vacuum interactions and that the vacuum field is in fact formally necessary for the stability of atoms in quantum theory. As summarized in an earlier paper addressing spontaneous

H. E. Puthoff $(\bowtie)$

Institute for Advanced Studies at Austin, 11855 Research Blvd, Austin, TX 78759, USA

e-mail: puthoff@aol.com; puthoff@earthtech.org 
emission processes: "The crucial role of the vacuum fluctuations emerges in the ground state of matter. The stability of the ground state (i.e., the fact that it does not radiate) is purely a quantum effect which is due to the vacuum fluctuations [1]."

Setting aside a full quantum mechanical treatment, it is sufficient for heuristic purposes to treat such problems semiclassically on the basis of point particles interacting with a random, classical radiation field whose spectral characteristics are those of the known quantum vacuum ZPF distribution, an approach known as stochastic electrodynamics (SED). Early detailed pedagogical papers by Boyer [2,3] provide a broad foundation for the SED approach as applicable to many problems generally considered to require quantum mechanical treatment. The further development of SED in the decades following is well-summarized in [4]. The treatment in this paper extends earlier work by the author on the quantum ground state of hydrogen [5] to address the case for quantum ground states in general. The SED ansatz takes advantage of the fact that SED derivations of vacuum-fluctuation-driven phenomena based on multipole/radiation-field interactions parallel Heisenberg-picture derivations in standard quantum electrodynamics (QED) $[6,7]$. Specifically, despite shortcomings in the SED approach as a proposed alternative to quantum theory [8], in the cases considered here calculations in SED are analogous to QED calculations with a symmetric ordering of photon creation and annihilation operators [9]. Before considering application on the basis of a general formalism, let us apply the central argument in detail to the simple one-dimensional harmonic oscillator.

\section{Nonrelativistic harmonic oscillator}

For a one-dimensional harmonic oscillator of natural frequency $\omega_{0}$ located at the origin $\mathbf{r}=0$ and immersed in the vacuum ZPF field, the (nonrelativistic) Abraham-Lorentz equation of motion for a point particle of mass $m$ and charge $q$, including radiation damping, is given by [10]

$m \ddot{x}+m \omega_{0}^{2} x=\left(\frac{q^{2}}{6 \pi \varepsilon_{0} c^{3}}\right) \dddot{x}+q E_{x}^{\mathrm{zp}}$,

where $E_{x}^{\mathrm{zp}}$ is the $x$ component of the vacuum ZPF electric field and here we neglect the force contribution from the magnetic field (see Sect. 3, however).

The required expression for the electric field is obtained as a component of the isotropic, homogeneous electromagnetic vacuum ZPF distribution whose spectral energy density is given by the Lorentz-invariant expression [7, p. 49; 11]

$\rho(\omega) \mathrm{d} \omega=\frac{\hbar \omega^{3}}{2 \pi^{2} c^{3}} \mathrm{~d} \omega$,

which corresponds to an energy $\hbar \omega / 2$ per normal mode. In the SED ansatz the Fourier composition underlying this spectrum can be written as a sum of plane waves

$\mathbf{E}^{\mathrm{zp}}=\operatorname{Re} \sum_{\sigma=1}^{2} \int d^{3} k \hat{\varepsilon} \sqrt{\frac{\hbar \omega}{8 \pi^{3} \varepsilon_{0}}} e^{i \mathbf{k} \cdot \mathbf{r}-i \omega t+i \theta(\mathbf{k}, \sigma)}$.

A similar expression for the magnetic field is obtained by replacing $\mathbf{E}^{\mathrm{zp}}$ by $\mathbf{H}^{\mathrm{zp}}, \hat{\varepsilon}$ by $(\hat{\mathbf{k}} \times \hat{\varepsilon})$, and $\varepsilon_{0}$ by $\mu_{0}$. In these expressions Re denotes 'Real part of,' $\sigma=1,2$ denote orthogonal polarizations, $\hat{\varepsilon}$ and $\hat{\mathbf{k}}$ are orthogonal unit vectors in the direction of the electric field polarization and wave propagation vectors, respectively, $\theta(\mathbf{k}, \sigma)$ are random phases distributed uniformly in the interval 0 to $2 \pi$ (independently distributed for each $\mathbf{k}, \sigma$ ), and $\omega=k c$.

Substitution of Eq. (3) into Eq. (1) leads to the following expressions for displacement, velocity and acceleration:

$x=\frac{q}{m} \operatorname{Re} \sum_{\sigma=1}^{2} \int d^{3} k(\hat{\varepsilon} \cdot \hat{\mathbf{x}}) \sqrt{\frac{\hbar \omega}{8 \pi^{3} \varepsilon_{0}}} \frac{1}{D} e^{i \mathbf{k} \cdot \mathbf{r}-i \omega t+i \theta(\mathbf{k}, \sigma)}$,

$v=\dot{x}=\frac{q}{m} \operatorname{Re} \sum_{\sigma=1}^{2} \int d^{3} k(\hat{\varepsilon} \cdot \hat{\mathbf{x}}) \sqrt{\frac{\hbar \omega}{8 \pi^{3} \varepsilon_{0}}}\left(-\frac{i \omega}{D}\right) e^{i \mathbf{k} \cdot \mathbf{r}-i \omega t+i \theta(\mathbf{k}, \sigma)}$, 
$a=\ddot{x}=\frac{q}{m} \operatorname{Re} \sum_{\sigma=1}^{2} \int d^{3} k(\hat{\varepsilon} \cdot \hat{\mathbf{x}}) \sqrt{\frac{\hbar \omega}{8 \pi^{3} \varepsilon_{0}}}\left(-\frac{\omega^{2}}{D}\right) e^{i \mathbf{k} \cdot \mathbf{r}-i \omega t+i \theta(\mathbf{k}, \sigma)}$,

where

$D=-\omega^{2}+\omega_{0}^{2}-i \Gamma \omega^{3}$,

$\Gamma=\frac{q^{2}}{6 \pi \varepsilon_{0} m c^{3}}$.

Now, for bounded, steady-state motion, we assume stationary expectation values for the mean-square position variable $x$ and its time derivatives. Thus,

$$
\begin{aligned}
\left\langle x^{2}\right\rangle= & \langle x \cdot x\rangle=\frac{q^{2}}{m^{2}} \sum_{\sigma=1}^{2} \sum_{\sigma^{\prime}=1}^{2} \int d^{3} k \int d^{3} k^{\prime}(\hat{\varepsilon} \cdot \hat{\mathbf{x}})\left(\varepsilon^{\prime} \cdot \mathbf{x}\right) \sqrt{\frac{\hbar \omega}{8 \pi^{3} \varepsilon_{0}} \sqrt{\frac{\hbar \omega^{\prime}}{8 \pi^{3} \varepsilon_{0}}} \frac{1}{D D^{\prime *}}} \\
& \times \frac{1}{2} \operatorname{Re}\left\langle\exp \left[i\left(\mathbf{k}-\mathbf{k}^{\prime}\right) \cdot \mathbf{r}-i\left(\omega-\omega^{\prime}\right) t+i \theta(\mathbf{k}, \sigma)-i \theta\left(\mathbf{k}^{\prime}, \sigma^{\prime}\right)\right]\right\rangle,
\end{aligned}
$$

where use of the complex conjugate and the notation $(1 / 2)$ Re derive from use of exponential notation. With $\int d^{3} k \rightarrow \int \mathrm{d} \Omega_{k} \int d k k^{2}$, and averaging over random phases

$\operatorname{Re}\left\langle\exp \left[i\left(\mathbf{k}-\mathbf{k}^{\prime}\right) \cdot \mathbf{r}-i\left(\omega-\omega^{\prime}\right) t+i \theta(\mathbf{k}, \sigma)-i \theta\left(\mathbf{k}^{\prime}, \sigma^{\prime}\right)\right]\right\rangle=\delta_{\sigma \sigma^{\prime}} \delta_{\omega \omega^{\prime}} \delta^{3}\left(\mathbf{k}-\mathbf{k}^{\prime}\right)$.

Equation (9) can, therefore, be simplified to

$$
\left\langle x^{2}\right\rangle=\frac{q^{2}}{2 m^{2}} \int \mathrm{d} \Omega_{k}\left[\sum_{\sigma=1}^{2}(\hat{\varepsilon} \cdot \hat{\mathbf{x}})^{2}\right] \int d k k^{2}\left[\frac{\hbar \omega}{8 \pi^{3} \varepsilon_{0}}\right] \frac{1}{D D^{*}} .
$$

We further note that, with the sum over polarizations given by

$$
\sum_{\sigma=1}^{2}\left[\hat{\varepsilon}(\mathbf{k}, \sigma) \cdot \hat{\mathbf{x}}_{i}\right]\left[\hat{\varepsilon}(\mathbf{k}, \sigma) \cdot \hat{\mathbf{x}}_{j}\right]=\delta_{i j}-\left(\hat{\mathbf{k}} \cdot \hat{\mathbf{x}}_{i}\right)\left(\hat{\mathbf{k}} \cdot \hat{\mathbf{x}}_{j}\right),
$$

the angular integration in $k$ takes the form

$$
\int \mathrm{d} \Omega_{k}\left[\sum_{\sigma=1}^{2}(\hat{\varepsilon} \cdot \hat{\mathbf{x}})^{2}\right]=\int \mathrm{d} \Omega_{k}\left[1-(\hat{\mathbf{k}} \cdot \hat{\mathbf{x}})^{2}\right]=\frac{8 \pi}{3} .
$$

Substitution of Eq. (13) into Eq. (11), and a change of variables to $\omega=k c$, then leads to

$$
\left\langle x^{2}\right\rangle=\frac{q^{2} \hbar}{6 \pi^{2} \varepsilon_{0} m^{2} c^{3}} \int_{0}^{\infty} \frac{\omega^{3} \mathrm{~d} \omega}{D D^{*}}=\frac{q^{2} \hbar}{6 \pi^{2} \varepsilon_{0} m^{2} c^{3}} \int_{0}^{\infty} \frac{\omega^{3} \mathrm{~d} \omega}{\left(-\omega^{2}+\omega_{0}^{2}\right)^{2}+\Gamma^{2} \omega^{6}} .
$$

Due to the smallness of $\Gamma$ for charge-to-mass ratios of interest the integrand in Eq. (14) is sharply peaked around $\omega=\omega_{0}$. We can, therefore, invoke the standard resonance approximation, extending the limits of integration and replacing $\omega$ by $\omega_{0}$ in all but the difference term. This yields, with substitution of the definition of $\Gamma$ from Eq. (8), the mean square fluctuation in position as

$$
\left\langle x^{2}\right\rangle=\frac{\hbar}{2 m \omega_{0}} \int_{-\infty}^{\infty} \frac{1}{\pi} \frac{\left(\Gamma \omega_{0}^{2} / 2\right) \mathrm{d} \omega}{\left(\omega_{0}-\omega\right)^{2}+\left(\Gamma \omega_{0}^{2} / 2\right)^{2}}=\frac{\hbar}{2 m \omega_{0}},
$$

since the (Lorentzian lineshape) integral is unity.

Calculation of the mean square fluctuation in velocity $\left\langle v^{2}\right\rangle=\left\langle\dot{x}^{2}\right\rangle$ follows as above, except that $\left(1 / D D^{*}\right) \rightarrow$ $(-i \omega / D)\left(i \omega / D^{*}\right)=\left(\omega^{2} / D D^{*}\right)$, yielding

$\left\langle v^{2}\right\rangle=\frac{\hbar \omega_{0}}{2 m}$. 
A similar calculation for the mean square fluctuation in acceleration $\left\langle a^{2}\right\rangle=\left\langle\ddot{x}^{2}\right\rangle$ yields

$\left\langle a^{2}\right\rangle=\frac{\hbar \omega_{0}^{3}}{2 m}$.

With the above calculations in hand we are now in a position to characterize the ground state of the harmonic oscillator. First, the mean square fluctuation in position given by Eq. (15) matches that obtained in the usual quantum mechanical treatment. Second, the mean square fluctuation in momentum, given by

$\left\langle p^{2}\right\rangle=m^{2}\left\langle v^{2}\right\rangle=\frac{m \hbar \omega_{0}}{2}$,

also matches that obtained from the standard QM treatment. The harmonic oscillator's ground state energy, kinetic plus potential, is given by

$\langle E\rangle=\frac{1}{2} m\left\langle v^{2}\right\rangle+\frac{1}{2} m \omega_{0}^{2}\left\langle x^{2}\right\rangle=\frac{\hbar \omega_{0}}{2}$,

also in agreement with the QM result.

Since the mean position $\langle x\rangle$ and mean momentum $\langle p\rangle$ of the stationary-state oscillator are zero, we also calculate the uncertainty relationship as

$\Delta x \Delta p=\sqrt{\left\langle(\Delta x)^{2}(\Delta p)^{2}\right\rangle}=\sqrt{\left\langle(x-\langle x\rangle)^{2}\right\rangle\left\langle(p-\langle p\rangle)^{2}\right\rangle}=\sqrt{\left\langle x^{2}\right\rangle\left\langle p^{2}\right\rangle}=\frac{\hbar}{2}$,

again in agreement with the known QM result.

Now, in accordance with the argument being pursued here, we compare the average power being absorbed from the vacuum fluctuation distribution with that radiated due to accelerated motion to determine their relative magnitudes. The power absorbed from the electric field due to the driving force $\mathbf{F}=q \mathbf{E}^{\mathrm{zp}}$ is given by

$\left\langle P_{\mathrm{abs}}\right\rangle=\langle\mathbf{F} \cdot \mathbf{v}\rangle=\left\langle q E_{x}^{\mathrm{zp}} \dot{x}\right\rangle$.

With $\mathbf{E}^{\text {zp }}$ and $\dot{x}$ given by Eqs. (3) and (5), respectively, the calculation carries through as in the derivation of $\left\langle x^{2}\right\rangle$ above to yield

$$
\left\langle P^{\mathrm{abs}}\right\rangle=\frac{q^{2} \hbar \omega_{0}^{3}}{12 \pi \varepsilon_{0} m c^{3}} .
$$

The power radiated due to accelerated motion is given by the standard Larmor expression as [12]

$$
\left\langle P^{\mathrm{rad}}\right\rangle=\frac{q^{2}\left\langle a^{2}\right\rangle}{6 \pi \varepsilon_{0} c^{3}}=\frac{q^{2}\left\langle\ddot{x}^{2}\right\rangle}{6 \pi \varepsilon_{0} c^{3}},
$$

which, with substitution from Eq. (17) and comparison with Eq. (22), yields

$$
\left\langle P^{\mathrm{rad}}\right\rangle=\left\langle P^{\mathrm{abs}}\right\rangle \text {. }
$$

Thus we find that the ground-state parameters of the quantum mechanical harmonic oscillator can be accounted for on the basis of interaction between a harmonically bound point particle and the vacuum electromagnetic ZPFs. Specifically, the stationary ground state thus established derives from an average balance of power between that absorbed from the vacuum fluctuations and that radiated due to accelerated motion [13]. It can be noted in passing that even as one asymptotically approaches the limit $q \rightarrow 0$ (uncharged oscillator) this outcome remains the same as $q^{2}$ cancels out in the $\left\langle P^{\mathrm{rad}}\right\rangle=\left\langle P^{\mathrm{abs}}\right\rangle$ relationship.

\section{Generalized approach}

Having derived the above relationship between absorbed and radiated powers for the harmonic oscillator's ground state, we now inquire as to whether this balance is specific to the harmonic oscillator by virtue of its simple linear 
restoring force, or can be extended to more general cases (e.g., nonlinear oscillator, hydrogen atom, particle in a box, etc.).

We begin with the generalization of Eq. (1)

$m \ddot{\mathbf{r}}=\Gamma m \dddot{\mathbf{r}}+q\left(\mathbf{E}^{\mathrm{zp}}+\dot{\mathbf{r}} \times \mathbf{B}^{\mathrm{zp}}\right)+\mathbf{F}^{\mathrm{ext}}$,

and we assume $\mathbf{F}^{\text {ext }}=-\nabla V$ for a broad class of cases of interest, with $V$ a time-independent confining potential. Multiplication of Eq. (25) by $\dot{\mathbf{r}}$, taking into account mathematical simplifications (e.g., $\dot{\mathbf{r}} \cdot\left(\dot{\mathbf{r}} \times \mathbf{B}^{\text {zp }}\right) \equiv$ $0, \quad(1 / 2) \mathrm{d} / \mathrm{d} t\left(\dot{\mathbf{r}}^{2}\right)=\dot{\mathbf{r}} \cdot \ddot{\mathbf{r}}, \mathrm{d} V / \mathrm{d} t=\partial V / \partial t+\dot{\mathbf{r}} \cdot \nabla \mathbf{V} \rightarrow \dot{\mathbf{r}} \cdot \nabla \mathbf{V}$ for a time-independent potential, etc.), followed by collection of terms, leads to

$\Gamma m\left\langle\ddot{\mathbf{r}}^{2}\right\rangle+\left\langle\frac{\mathrm{d}}{\mathrm{d} t}\left(\frac{1}{2} m \dot{\mathbf{r}}^{2}+V-\Gamma m \dot{\mathbf{r}} \cdot \ddot{\mathbf{r}}\right)\right\rangle=q\left\langle\dot{\mathbf{r}} \cdot \mathbf{E}^{\mathrm{zp}}\right\rangle$.

At this point we recall the earlier-quoted statement that derives from quantum theory: "The crucial role of the vacuum fluctuations emerges in the ground state of matter. The stability of the ground state (i.e., the fact that it does not radiate) is purely a quantum effect which is due to the vacuum fluctuations [1]." In the SED development presented here we find its expression in positing that application of (26) to represent the (by definition) stationary ground state perforce requires that the time-dependent second term on the lefthand side must be conjectured to vanish. Otherwise (26) could not be taken to represent a stable, time-independent ground state. ${ }^{1}$ As a consequence the remaining terms constitute the condition that reveals itself to be that the average power radiated due to accelerated motion (Larmor radiation) is balanced by the average power absorbed from the vacuum fluctuations. Substituting the definition of $\Gamma$ from Eq. (8) we obtain

$\frac{q^{2}}{6 \pi \varepsilon_{0} c^{3}}\left\langle a^{2}\right\rangle=\left\langle q \mathbf{E}^{\mathrm{zp}} \cdot \mathbf{v}\right\rangle$.

Thus the stationary ground state, although (from a classical viewpoint) involving accelerated charged-particle motion and hence possessing an associated Larmor radiation loss, is nonetheless observed to be overall radiationless in nature due to a compensatory absorption from the background electromagnetic vacuum ZPFs. The balance so obtained also accounts for the well-known fact that an oscillator or atom in its ground state does not on net absorb zero-point radiation and, therefore, remains in its ground state. Finally, we note that this general result is independent of the form of the (time-independent) confining potential $V$ and is thus applicable to a wide range of problems.

\section{Concluding remarks}

Addressed is the seeming paradox that even though quantum ground states typically involve charged particles in accelerated motions, such states are nonetheless observed to be radiationless in nature. Though this feature is overlooked in everyday application of quantum theory to ground-state description, nonetheless this remarkable property is worthy of some discussion and clarification. In detail, it is the recognition that ground-state atomic structures are not isolated entities in an empty background, but are perforce immersed in a background of vacuum fluctuations. With regard to the behavior of charged particles, the primary component of interest is that of the vacuum electromagnetic ZPFs. Atoms (and other quantum systems), therefore, constitute open systems engaged in dynamic interactions with the underlying vacuum states. Specifically, the on net radiationless characteristic of the ground state is seen to derive from particle-vacuum interactions in which a dynamic equilibrium is established between radiation emission due to particle acceleration, and compensatory absorption from the ZPFs of the vacuum

\footnotetext{
${ }^{1}$ For the trivial case of a perfectly circular orbit this term vanishes even before averaging, given that the kinetic and potential energies are constant and the velocity and acceleration vectors are orthogonal. Beyond that, SED modeling attempts involving lengthy numerical simulations for hydrogenic atoms to verify the vanishing of the time-dependent term on the lefthand side of (26) have to date only been marginally successful, self-ionization of the atom often being the outcome [14,15]. Under consideration are the incorporation of additional factors such as expansion beyond the dipole approximation, spin-orbit coupling, relativistic effects and so forth; thus simulation studies remain a work in progress.
} 
electromagnetic field. ${ }^{2}$ Thus, employing an SED approach, we have shown here in detail that, as argued in [1], the vacuum field is formally necessary for the stability of atomic structures, and this underlying principle, therefore, constitutes an important feature of quantum ground states.

Open Access This article is distributed under the terms of the Creative Commons Attribution 4.0 International License (http:// creativecommons.org/licenses/by/4.0/), which permits unrestricted use, distribution, and reproduction in any medium, provided you give appropriate credit to the original author(s) and the source, provide a link to the Creative Commons license, and indicate if changes were made.

\section{References}

1. Fain, B.: Spontaneous emission vs. vacuum fluctuations. Il Nuovo Cimento B 68, 73-78 (1982)

2. Boyer, T.H.: Random electrodynamics: the theory of classical electrodynamics with classical electromagnetic zero-point radiation. Phys. Rev. D 11, 790-808 (1975)

3. Boyer, T.H.: General connection between random electrodynamics and quantum electrodynamics for free electromagnetic fields and for dipole oscillator systems. Phys. Rev. D 11, 809-830 (1975)

4. de la Pena, L., Cetto, A.M.: The Quantum Dice: An introduction to Stochastic Electrodynamics. Kluwer Academic Publisher, Dordrecht (1996)

5. Puthoff, H.E.: Ground state of hydrogen as a zero-point-fluctuation-determined state. Phys. Rev. D 35, 3266-3269 (1987)

6. Milonni, P.W.: Semiclassical and quantum electrodynamical approaches in nonrelativistic radiation theory. Phys. Rep. 25, 1-81 (1976)

7. Milonni, P.W.: The Quantum Vacuum. Academic Press, Boston (1994). (Sect. 8.12)

8. Davis, E.W., Puthoff, H.E.: On extracting energy from the quantum vacuum. In: Millis, M.G., Davis, E.W. (eds.) Frontiers of Propulsion Science, pp. 569-603. American Institute of Aeronautics and Astronautics, Inc., Reston (2009)

9. Milonni, P.W., Smith, W.A.: Radiation reaction and vacuum fluctuations in spontaneous emission. Phys. Rev. A 11, 814-824 (1975)

10. Jackson, J.D.: Classical Electrodynamics, 2nd edn. Wiley, New York (1975)

11. Boyer, T.H.: Derivation of the blackbody radiation spectrum without quantum assumptions. Phys. Rev. 182, 1374-1383 (1969)

12. Feynman, R.P., Leighton, R.B., Sands, M.: The Feynman Lectures on Physics, vol. 1, p. 32-2. Addison-Wesley, Reading (1963)

13. Senitzky, I.R.: Dissipation in quantum mechanics: the quantum oscillator. Phys. Rev. 119, 670-679 (1960)

14. Cole, D.C., Zou, Y.: Quantum mechanical ground state of hydrogen obtained from classical electrodynamics. Phys. Lett. A 317, 14-20 (2003)

15. Nieuwenhuizen, T.M., Liska, M.T.P.: Simulation of the hydrogen ground state in stochastic electrodynamics. arXiv: $1502.06856 \mathrm{v} 1$ [quant-ph] (24 Feb 2015)

16. de la Pena, L., Cetto, A.M., Hernandez, A.V.: The Emerging Quantum: The Physics Behind Quantum Mechanics. Springer, New York (2015)

2 Further development of the foundational nature of the radiationless state for quantum mechanics in general can be found in [16], which is an update to the material presented in [4]. In this later compendium additional constraints on the SED approach are incorporated to match more closely the requirements of QED (e.g., detailed balance of energy). In the updated approach, labeled LSED (linear SED), it remains the case that "the ZPF is seen as the source of the quantum behavior of matter." 